\title{
Heart Block and Coronary Artery Disease
}

\author{
M. J. DAVIES, * M.B., B.S. ; D. REDWOOD, † M.A., M.B., B.CHIR., M.R.C.P. ; A. HARRIS, † M.B., B.S., M.R.C.P.
}

Brit. med. F., 1967, 3, 342-343

Acute heart block is an uncommon but serious complication of myocardial infarction, though survivors usually return to sinus rhythm.

Chronic heart block associated with Stokes-Adams attacks is also often assumed to be ischaemic in origin. Some clinical reviews have, however, stressed the high incidence of "primary" heart block (Zoob and Smith, 1963; Johansson, 1966). Initial studies from this hospital suggested that significant coronary artery disease was not present in most cases of chronic heart block and that pathological changes were often confined to the conduction system (Portal et al., 1962).

A detailed post-mortem study of both acute and chronic heart block has now been carried out to define the part played by coronary disease in the production of complete heart block.

\section{Material and Methods}

The material comprised the hearts from 14 cases of atrioventricular $(\mathrm{A}-\mathrm{V})$ block associated with acute myocardial infarction and 53 cases of chronic complete heart block. Many of the hearts from cases of acute infarction were received as post-mortem specimens from other hospitals. The majority of cases of chronic heart block had Stokes-Adams attacks and had been referred to this hospital for pacing. The average age at death for this group was 62. A control series of 35 hearts obtained from routine necropsy material was studied in an identical manner. The control subjects were over the age of 50 at the time of death and had had a normal electrocardiogram in the last three months of life.

Post-mortem examination of the hearts included coronary angiography (Crawford et al., 1961) and serial sections of the coronary arteries at 5-mm. intervals. The degree of atherosclerosis in the coronary arteries was assessed by scoring the degree of stenosis in each of 16 segments, after the method of Eusterman et al. (1962). The entire conduction system was studied by use of a serial histological section technique (Davies, 1967).

\section{Results}

Acute Infarction.-In 9 of the 14 cases of acute infarction there was thrombotic occlusion of a dominant right coronary artery which supplied the posterior wall of the heart and ultimately the $A-V$ node. In four cases there was occlusion of a dominant left circumflex artery supplying the posterior wall of the heart and A-V node. These 13 cases showed recent posteroseptal infarction. In the remaining patient there was occlusion of the anterior descending coronary artery with a large anteroseptal infarct involving both bundle branches. The site of damage to the conduction system in acute infarction is shown in Table I. Pathological changes in the conduction system with the usual posteroseptal infarct were often relatively minor. If major damage occurred the changes were found in the bundle branches.

2. Chronic Heart Block.-The lesions in the conduction system responsible for chronic heart block in 53 cases are shown

\footnotetext{
* Department of Pathology, St. George's Hospital Medical School, London S.W.1.

t Department of Cardiology, St. George's Hospital Medical School,
London S.W.1.
}

in Table II. Coronary disease was the cause in only eight and was associated with destruction of both bundle branches in the scarring of old septal infarction. In five of the eight cases this was posteroseptal and in three anteroseptal. Severe atherosclerosis was present in all cases, and recanalized or old occlusions were demonstrable in the coronary arteries in relation to the areas of old infarction. The commonest cause of chronic complete heart block was bundle-branch fibrosis, which accounted for 21 cases. Loss of conduction fibres had occurred in the upper two-thirds of both bundle branches. The immediately adjacent myocardium was normal. The coronary arteries in this group showed no significant degree of atheroma and did not differ from the control group when scored for coronary artery disease, the method devised by Eusterman et al. (1962), with serial (5-mm.) sections of the coronary arteries, being used. Focal myocardial scarring was increased compared with the controls, but major pathological changes were more or less confined to the conduction system.

TABLE I.-Pathology of the Conduction System in 14 Cases of Acute

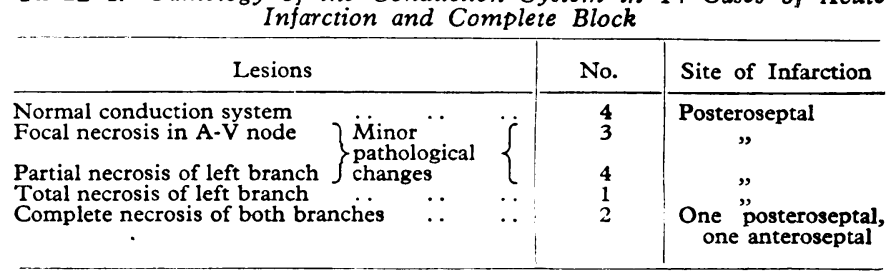

TABLE II.-Aetiology of 53 Cases of Chronic A-V Block at Necropsy

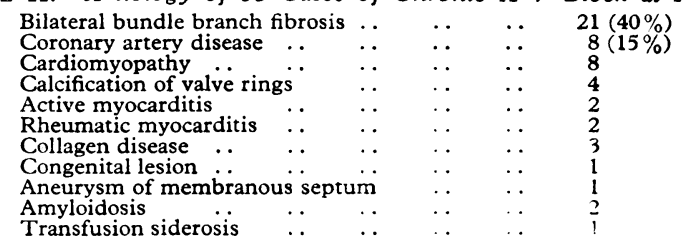

\section{Discussion}

This paper is concerned only with the relation of coronary artery disease to complete heart block. Detailed analysis of other and more common causes of heart block are reported elsewhere (Davies, 1967).

In the majority of patients who develop complete heart block as a complication of myocardial infarction the conduction disturbance is transitory. This feature has been stressed in clinical reviews, and few survivors need long-term pacing (Penton et al., 1956; Gilchrist, 1958 ; Harris and Bluestone, 1966). Even in those who die major structural damage to the conduction system is found in only a minority. In most cases it is therefore reasonable to presume that heart block is due to anoxia of the $A-V$ node secondary to an occlusion of the dominant coronary artery, usually the right, which supplies the node. In a minority with acute infarction major damage is found to involve the bundle branches, and only if both branches are totally destroyed will block be permanent.

In the eight cases of chronic heart block due to coronary artery disease, comprising only $15 \%$ of those with chronic $\mathrm{A}-\mathrm{V}$ block, there was destruction of both bundle branches in 
areas of old infarction. This figure is in close agreement with that of Johansson (1966), who, on clinical criteria, suspected that only 24 out of 142 cases $(17 \%)$ of chronic heart block were ischaemic in origin. He offered no pathological explanation for 64 out of 142 cases ( $45 \%$ ) which were classified as "primary" heart block. In the present post-mortem series 21 out of 53 cases ( $40 \%$ ) were due to bundle branch fibrosis. The pathological basis of primary heart block is therefore bilateral bundle-branch fibrosis ; the cause remains unknown, but it is certainly not due to coronary artery disease.

\section{Summary}

In a post-mortem study of 53 hearts from patients with chronic atrioventricular block only $8(15 \%)$ were due to coronary artery disease. Bundle-branch fibrosis of unknown aetiology, without major damage to the contractile myocardium, is the commonest cause, being present in 21 cases (40\%).
In 14 cases of acute myocardial infarction and $\mathrm{A}-\mathrm{V}$ block major structural damage to the conduction system was unusual, but, when present, involved the bundle branches.

This work was done during tenure of the William Shepherd Research Fellowship from the Board of Governors of St. George's Hospital. We would like to thank Dr. A. Leatham and Dr. W. B. Robertson for their help in preparing this paper.

\section{REFERENCES}

Crawford, T., Dexter, D., and Teare, R. D. (1961). Lancet, 1, 181 Davies, M. J. (1967). f. Path. Bact. In press.

Eusterman, J. H., Achor, R. W. P., Kincaid, O. W., and Brown, A. L., jun. (1962). Circulation, 26, 1288.

Gilchrist, A. R. (1958). Scot. med. ₹., 3, 53.

Gilchrist, A. R. (1958). Scot. med. F., 3, 53. Heart f., 28, 631.

Harris, A., and Bluestone, R. (1966). Brit. Heart f., 28, 631. 451.

Penton, G. B., Miller, H., and Levine, S. A.'(1956). Circulation, 13, 801. Portal, R. W., Davies, J. G., Leatham, A., and Siddons, A. H. M. (1962). Lancet, 2, 1369.

Zoob, M., and Smith, K. S. (1963). Brit. med. F., 2, 1149.

\title{
Dip-Slide : An Aid to Quantitative Urine Culture in General Practice
}

\author{
DENNIS GUTTMANN,* M.A., M.B., B.CH., B.SC., M.R.C.P.; G. R. E. NAYLOR, $†$ M.A., M.D., M.R.C.P., M.C.PATH.
}

Brit. med. F., 1967, 3, 343-345

Since Kass (1956) defined criteria of significant bacteriuria, the use of quantitative assessments of the bacterial population as an aid to the differentiation between infection and contamination of the urine has gained wide acceptance. The pour-plate method is recognized as the standard technique for performing viable counts on urine, but requires trained staff for setting up the cultures. If misleading results are to be avoided, urine specimens must be cultured within an hour or two of collection, or be refrigerated; in general practice it is often difficult to fulfil these requirements. Further, the examination of large numbers of specimens, as at antenatal clinics and in population surveys, makes demands on laboratory services which may become prohibitive if the pour-plate method is employed routinely. A number of simpler cultural methods have been described, such as the filter-paper strip inoculation technique of Leigh and Williams (1964), and the semiquantitative methods of Cattell and Lefford (1963) and of Guttmann and Stokes (1963). Though these are technically less complicated, they are still dependent on the delivery to the laboratory of freshly passed specimens.

Mackey and Sandys (1965, 1966) described a dip-inoculum transport medium in which a metal or plastic spoon carries the culture medium, and which may be inoculated by simply dipping in the specimen, after which it need not reach the laboratory for incubation for 24 or 48 hours. Using standard materials readily available in most laboratories, we have investigated a similar method which we feel has a number of advantages. An ordinary $3-$ by 1 -in. ( $7.5-$ by $2.5-\mathrm{cm}$.) glass microscope slide is coated for a length of about 2 in. $(5 \mathrm{~cm}$.) with nutrient agar on one side and with MacConkey's agar on the other. It is held in a small cylindrical aluminium container as supplied in the Public Health Laboratory Service for the collection of faeces specimens. This dip-slide is inoculated by dipping the coated end into the fresh urine specimen. After allowing excess urine to drain for a few seconds, the slide is returned to its container and after replacing the screw cap it is sent to the

* Addenbrooke's Hospital, Cambridge.

t Public Health Laboratory Service, Cambridge. laboratory, if necessary by post. After overnight incubation the number of colonies which have grown on the slide is counted with a hand lens. The preparation is cheap enough to be discarded after use, and an extensive trial has shown the method to give clinically valuable results. Fig. 1 shows the dip-slide and its aluminium container. Full details of the preparation of the dip-slides are given elsewhere (Naylor and Guttmann, 1967).

Dilutions of broth cultures of Gram-negative rods and Grampositive cocci isolated from pyogenic infections of the urinary tract were examined by dip-slide inoculation and simultaneous viable bacterial counts: we found a straight-line relationship between the number of colonies on the dip-slide and the number of organisms in the fluid into which it had been dipped ; 50 to 100 colonies on the dip-slide correspond to a viable bacterial count of 10,000 per ml. (Naylor and Guttmann, 1967). Fig. 2 shows a series of dip-slides prepared from dilutions of a culture of Escherichia coli, and it will be seen that separate colonies readily countable with a hand lens were obtained up to a concentration of $0.6 \times 10^{5}$ organisms per $\mathrm{ml}$. The results of a clinical trial are reported in more detail below.

\section{Methods}

From outpatients attending a urinary infection clinic 385 consecutive mid-stream urine specimens were obtained and examined in parallel by the dip-slide, a pour-plate viable count, and the semiquantitative method of Guttmann and Stokes (1963). The last 100 specimens were inoculated on to dip-slides with cycloheximide added to the nutrient agar at a concentration of $100 \mu \mathrm{g} . / \mathrm{ml}$. of medium, as well as on to ordinary dipslides. Cycloheximide prevents the growth of air-borne mould spores which occasionally were found to contaminate some of the slides during preparation, thus creating difficulties if the dip-slides were stored for some weeks before use. Such a concentration of cycloheximide did not interfere with the growth of bacteria isolated from urine specimens.

Microscopy of the Gram-stained deposit and tests for protein were performed, and white cell excretion rates were estimated by 